\title{
TU/e EmonOWEN

\section{Verification of hypertorus communication grids by infinite Petri nets and process algebra}

Citation for published version (APA):

Zaitsev, D., Shmeleva, T., \& Groote, J. F. (2019). Verification of hypertorus communication grids by infinite Petri nets and process algebra. IEEE/CAA Journal of Automatica Sinica, 6(3), 733-742. [8707130].

https://doi.org/10.1109/JAS.2019.1911486

\section{Document license:}

TAVERNE

DOI:

10.1109/JAS.2019.1911486

Document status and date:

Published: 01/05/2019

\section{Document Version:}

Publisher's PDF, also known as Version of Record (includes final page, issue and volume numbers)

\section{Please check the document version of this publication:}

- A submitted manuscript is the version of the article upon submission and before peer-review. There can be important differences between the submitted version and the official published version of record. People interested in the research are advised to contact the author for the final version of the publication, or visit the $\mathrm{DOI}$ to the publisher's website.

- The final author version and the galley proof are versions of the publication after peer review.

- The final published version features the final layout of the paper including the volume, issue and page numbers.

Link to publication

\section{General rights}

Copyright and moral rights for the publications made accessible in the public portal are retained by the authors and/or other copyright owners and it is a condition of accessing publications that users recognise and abide by the legal requirements associated with these rights.

- Users may download and print one copy of any publication from the public portal for the purpose of private study or research.

- You may not further distribute the material or use it for any profit-making activity or commercial gain

- You may freely distribute the URL identifying the publication in the public portal.

If the publication is distributed under the terms of Article 25fa of the Dutch Copyright Act, indicated by the "Taverne" license above, please follow below link for the End User Agreement:

www.tue.nl/taverne

Take down policy

If you believe that this document breaches copyright please contact us at:

openaccess@tue.nl

providing details and we will investigate your claim. 


\title{
Verification of Hypertorus Communication Grids by Infinite Petri Nets and Process Algebra
}

\author{
Dmitry A. Zaitsev, Senior Member, IEEE, Tatiana R. Shmeleva, and Jan Friso Groote
}

\begin{abstract}
A model of a hypertorus communication grid has been constructed in the form of an infinite Petri net. A grid cell represents either a packet switching device or a bioplast cell. A parametric expression is obtained to allow a finite specification of an infinite Petri net. To prove properties of an ideal communication protocol, we derive an infinite Diophantine system of equations from it, which is subsequently solved. Then we present the programs htgen and ht-mcrl2-gen, developed in the $\mathrm{C}$ language, which generate Petri net and process algebra models of a hypertorus with a given number of dimensions and grid size. These are the inputs for the respective modeling tools Tina and mCRL2, which provide model visualization, step simulation, state space generation and reduction, and structural analysis techniques. Benchmarks to compare the two approaches are obtained. An ad-hoc induction-like technique on invariants, obtained for a series of generated models, allows the calculation of a solution of the Diophantine system in a parametric form. It is proven that the basic solutions of the infinite system have been found and that the infinite Petri net is bounded and conservative. Some remarks regarding liveness and liveness enforcing techniques are also presented.
\end{abstract}

Index Terms-Computing grid, conservativeness, deadlock, hypertorus, infinite Petri nets, process algebra, systems biology.

\section{INTRODUCTION}

$\mathbf{H}$ YPERCUBE Petri net models [1]-[5] are suited for parallel and distributed computer systems [6], [7], computing grids [7], and systems biology [8]. The nature of a cell is different, but the structures of triangular, square, and hexagonal grids on a plane or a hypercube for a greater number of dimensions, look alike. Communication devices and cells have similar behavior: a computing or communication device transmits packets, while a bioplast cell transmits substances and impulses. In [6] it is stated that a torus network is the best topology for supercomputing and that using such networks decreases communication overhead considerably; the majority

Manuscript received November 8, 2018; revised January 13, 2019; accepted January 20, 2019. This work was supported in part by NATO (ICS.NUKR.CLG982689). Recommended by Associate Editor MengChu Zhou. (Corresponding author: Dmitry A. Zaitsev.)

Citation: D. A. Zaitsev, T. R. Shmeleva, and J. F. Groote, "Verification of hypertorus communication grids by infinite petri nets and process algebra," IEEE/CAA J. Autom. Sinica, vol. 6, no. 3, pp. 733-742, May 2019.

D. A. Zaitsev is with the Vistula University, Warsaw 02-787, Poland (email: daze@acm.org).

T. R. Shmeleva is with the A.S. Popov Odessa National Academy of Telecommunications, Odessa 65023, Ukraine (e-mail: t.shmeleva@onat.edu.ua).

J. F. Groote is with the Eindhoven University of Technology, PO Box 513, 5600 MB Eindhoven, The Netherlands (e-mail: j.f.groote@tue.nl).

Color versions of one or more of the figures in this paper are available online at http://ieeexplore.ieee.org.

Digital Object Identifier 10.1109/JAS.2019.1911486 of the top supercomputers, including IBM Blue Gene/Q, use a multidimensional torus.

Open grids with pending contact places represent auxiliary constructs. For modeling real-life systems, certain edge conditions are specified. Among various studied edge conditions [1]-[5] we choose a simple yet powerful condition that produces a hypertorus by closing the hypercube in each dimension and connecting its opposite edges. The (hyper)torus plays a key role in applications of nuclear physics [9], [10] and represents a convenient construct for modeling the human brain. It is a closed system where each cell has many connections. Greater numbers of dimensions allow one to represent more connections; the regularity of a structure gives the possibility of imposing any network of connections among cells similar to programmable logic matrices. The cell model could be easily replaced while the model structure remains the same.

The principles of generating Petri net models in the logical and graphical format of Tina [11], [12] are described in [2] for models of open square planar grids. In this article, we describe a program that generates hypertorus models in $\mathrm{d}$ dimensional space [13]. Its applications allow one to obtain the basic solutions of infinite linear Diophantine systems and then prove properties of ideal communication protocols for hypertorus grids.

The results are double checked with hypertorus grid models using process algebra constructed in the modeling system mCRL2 [14]-[16]. Obtained benchmarks are compared and the benefits of a combined approach are discussed.

The remainder of this paper is organized as follows: Section II defines a hypertorus grid model; Section III considers computation of place invariants of the grid model in a parametric form; Section IV describes a program htgen that is used for composing the parametric solutions of the infinite system of linear equations for calculating place invariants; in Section $\mathrm{V}$ a hypertorus grid model in mCRL2 is constructed and a program ht-mcrl2-gen that generates these models is described; in Section VI, benchmarking is described, and the benchmarks obtained for Tina and mCRL2 are compared.

\section{Composition of A Hypertorus Grid Model}

A cell model [1]-[5] represents a packet switching device working in a full-duplex mode based on a store-andforward principle. From a spatial point of view it is a unitsize hypercube in a $d$-dimensional space denoted as $H_{d}$ that consists of an internal buffer of packets and ports situated on the facets of the hypercube, which correspond to the VonNeumann neighborhood. Below we use the following notation 
for variables. The first character ' $p$ ' means a place and the first character ' $t$ ' means a transition. The second character specifies whether it is an input (' $i$ ') or an output (' $o$ '), while the character ' $b$ ' indicates that it is an internal buffer. The third letter ' $l$ ' specifies the limitation on buffer capacity. The following expression completely specifies $H_{d}$.

$$
\left(\begin{array}{c}
\left(\begin{array}{c}
\left.t o_{j, n}: p b_{j, n}, p o l_{j, n} \rightarrow p o_{j, n}, p b l\right), \\
\left(t i_{j, n, j^{\prime}, n^{\prime}}: p i_{j, n}, p b l \rightarrow p b_{j^{\prime}, n^{\prime}}, p i l_{j, n}\right)
\end{array}\right): \\
1 \leq j \leq d, 1 \leq n \leq 2, \\
1 \leq j^{\prime} \leq d, 1 \leq n^{\prime} \leq 2,\left(j^{\prime}, n^{\prime}\right) \neq(j, n) .
\end{array}\right)
$$

In (1), the input places of a transition are listed to the left of the " $\rightarrow$ " symbol. Its output places are listed after the " $\rightarrow$ " symbol. For indexed elements, the ranges of the indices are specified using parameters. Equation (1) has a single parameter $d$ equal to the number of dimensions.

Places $p b_{j, n}$ and $p b l$ model the internal buffers and the limitation on its size, respectively; transitions $t o_{j, n}$ specify the output tracts of ports while transitions $t i_{j, n, j^{\prime}, n^{\prime}}$ specify the input tracts implementing the packet forwarding decision; contact places $p o_{j, n}\left(p o l_{j, n}\right)$ and $p i_{j, n}\left(p i l_{j, n}\right)$ correspond to the port input and output buffers (limitation of their sizes), respectively. Ports, situated on the hypercube facet, are indexed by a pair of numbers $j$ and $n$ where $j$ denotes the dimension the port facet is perpendicular to and $n$ enumerates the corresponding two facets in the following way: value 1 corresponds to a facet which is closer to the origin and value 2 corresponds to a facet which is distant from the origin (on the coordinate $j$ ). We use a pair of indices $j^{\prime}$ and $n^{\prime}$ to specify the ports where a packet can be forwarded. Since the lower indices specify elements inside a cell and the upper indices specify the cells order within a grid, we use the multi-character notation of variables for clarity and compatibility with [1]-[5].

An example of a cell model for a 2-dimensional case is represented in Fig. 1. The grid composition is implemented via the merging of contact places of neighboring cells as described in [1]-[5]. An example grid obtained as a result of the composition for a 2-dimensional case is represented in Fig. 2.

A general hypertorus model in a $d$-dimensional space of size $k$ is denoted as $H T_{d, k}$ and specified by expression (2) where an additional parameter $k_{j}$ equals to the hypertorus size within dimension $j$. In software implementations we suppose for simplicity that $k_{1}=k_{2}=\cdots=k_{d}=k$.

$$
\begin{aligned}
& \left(\begin{array}{c}
\left(t o_{j, 1}^{\mathbf{i}}: p b_{j, 1}^{\mathbf{i}}, p o l_{j, 1}^{\mathbf{i}} \rightarrow p o_{j, 1}^{\mathbf{i}}, p b l^{\mathbf{i}}\right) \\
\left(t i_{j, 1, j^{\prime}, n^{\prime}}^{\mathbf{i}}: p i_{j, 1}^{\mathbf{i}}, p b l^{\mathbf{i}} \rightarrow p b_{j^{\prime}, n^{\prime}}^{\mathbf{i}}, p i l_{j, 1}^{\mathbf{i}}\right) \\
\left(t o_{j, 2}^{\mathbf{i}}: p b_{j, 2}^{\mathbf{i}}, p i l_{j, 1}^{x i(\mathbf{i}, j)} \rightarrow p i_{j, 1}^{x i(\mathbf{i}, j)}, p b l^{\mathbf{i}}\right) \\
\left(t i_{j, 2, j^{\prime}, n^{\prime}}^{\mathbf{i}}: p o_{j, 1}^{x i(\mathbf{i}, j)}, p b l^{\mathbf{i}} \rightarrow p b_{j^{\prime}, n^{\prime}}^{\mathbf{i}}, p o l_{j, 1}^{x i(\mathbf{i}, j)}\right)
\end{array}\right): \\
& x i(\mathbf{i}, j)=\mathbf{i}^{\prime}, \quad i_{l}^{\prime}=\left\{\begin{array}{c}
i_{l}, l \neq j, \\
x i j\left(i_{l}, j\right), l=j .
\end{array}\right. \\
& \operatorname{xij}(i, j)=\left\{\begin{array}{c}
i+1, i<k_{j}, \\
1, i=k_{j} .
\end{array}\right.
\end{aligned}
$$

where its initial marking is specified by

$$
\left(\begin{array}{c}
\left(\begin{array}{c}
\left(c * p b_{j, n}^{\mathbf{i}}, b * p b l^{\mathbf{i}}\right): 1 \leq n \leq 2, \\
\left(p i l_{j, n}^{\mathbf{i}}, p o l_{j, n}^{\mathbf{i}}\right): n=1
\end{array}\right): \\
\mathbf{i}=\left(i_{1}, \ldots, i_{d}\right), 1 \leq i_{j} \leq k_{j}, 1 \leq j \leq d
\end{array}\right)
$$

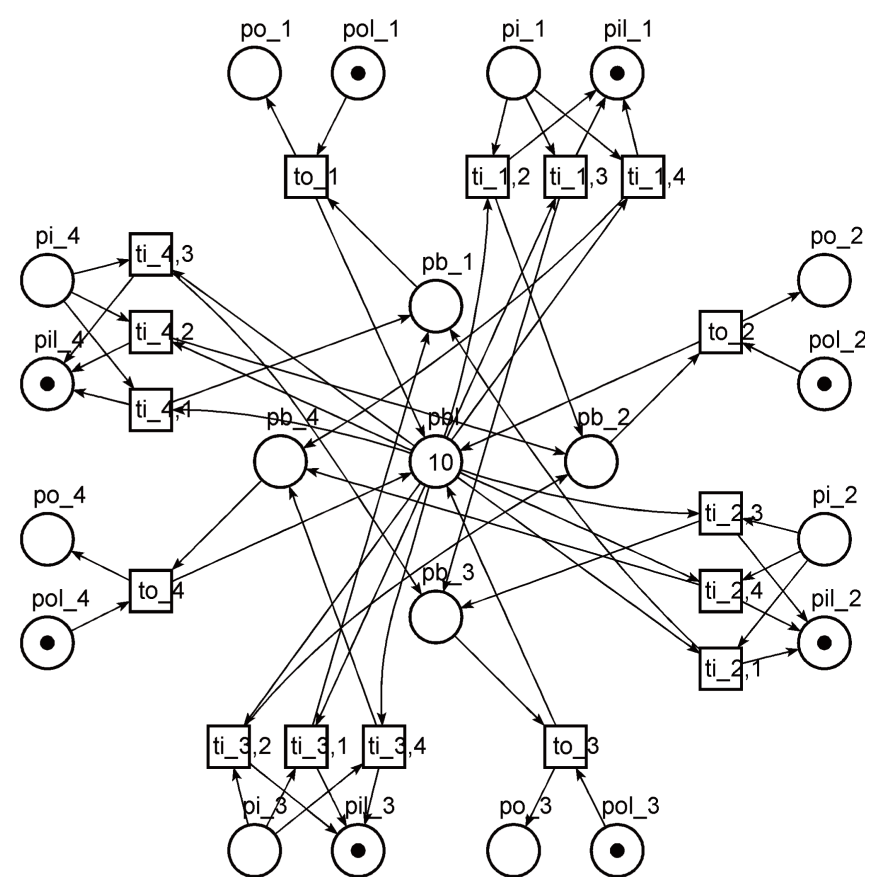

Fig. 1. Model of a cell in $\mathrm{H}_{2}$.

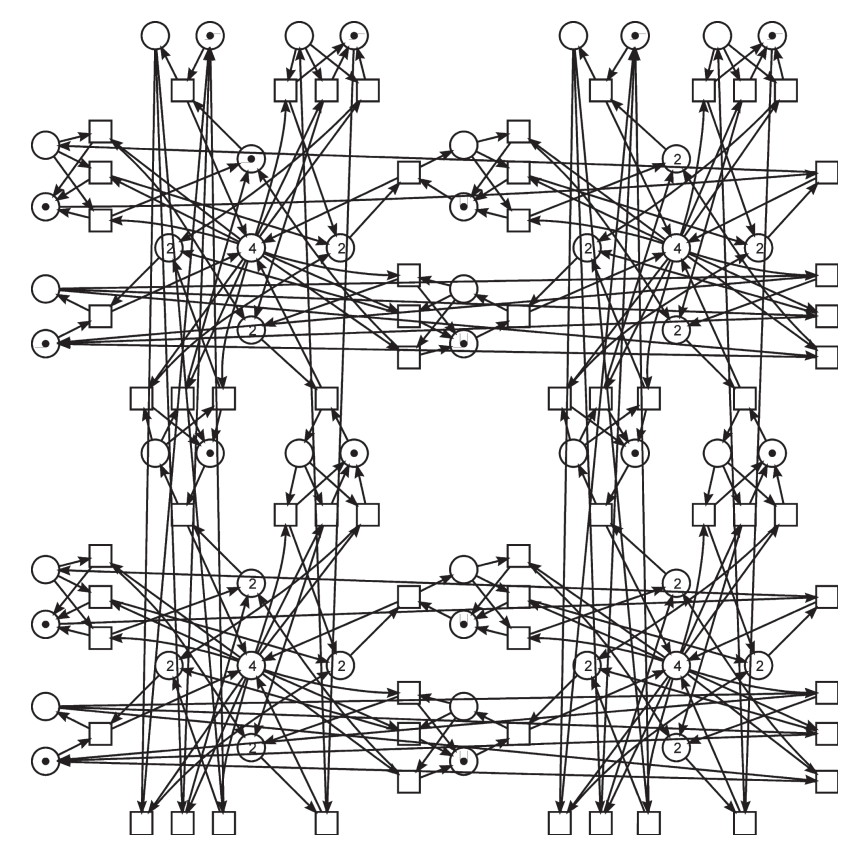

Fig. 2. Composition of a 2-dimensional structure $H T_{2,2}$.

In the initial marking, there are $c$ packets in each buffer section and the remaining available buffer size is equal to $b$. The notation is compatible with data formats of Tina [11], [12] where only nonzero magnitudes are listed and $v * p$ means that the value for place $p$ equals to $v$, where the unit values are omitted. 
Compared to hypercube models [2], [3], Equation (2) contains the following functions: function $x i(i, j)$ defines the index of the next cell in the Von-Neumann neighborhood with only one coordinate changed; function $x i j(i, j)$ defines the next cell for the number $j$ of the coordinate axis and supposes an increment for all values with exception of value $k_{j}$, for which it closes the (hyper) torus surface returning 1 . Generalized neighborhoods [17] are considered as a future research direction.

\section{ANALYSIS OF HYPERTORUS GRID MODEL}

For finite Petri nets, which represent models of either artificial or natural systems, three basic properties are the most significant: boundedness, conservativeness, and liveness [18], [19]. A live Petri net never halts, and in any state, each of its transitions can be enabled after a finite number of transition firings. The marking of a bounded net is limited by a certain constant; a conservative net preserves the (weighted) number of tokens during the evolution of a net.

Two basic techniques are employed for the analysis of Petri nets [20]: 1) the generation of the complete state space represented with a reachability graph and 2) the solution of linear equations and inequalities. The first approach allows investigating all the properties for bounded Petri nets, but the state space grows exponentially. The second approach provides a more efficient way to solve certain tasks, especially in subclasses of Petri nets [21]. Two auxiliary techniques, named decomposition and reduction, can help one to deal with exponential complexity. Decomposition [20]-[23] divides a net into a few subnets, which allows for conclusions on the properties of the entire net based on properties of its subnets, and reduction compresses a net while preserving its properties [20], [24], [25].

In Fig. 3 and Fig. 4, we give two examples of a state space for a 2-dimensional case and a grid of size 1. In Fig. 3, we have only one token simulating a packet, and in Fig. 4 we have 4 tokens simulating packets. In both cases, the additional buffer size equals zero. For Fig. 3, we obtain the state space of 8 states and 16 transitions, which exhibits a cubic form. For Fig. 4, we obtain 192 states and 1,008 transitions and its graphical representation looks rather tangled. For analogous initial markings in a 3-D space, we obtain the following states/transitions pairs: $12 / 36$ and 5336/60588, respectively. Note that example grids contain a single square and cube; for a planar grid of size $2 \times 2$, the number of states is estimated to be $10^{11}$ with Tina's experimental sift tool [11], [12]. Constructs for representing a state space of an infinite Petri net is considered as a future research direction.

Solving the systems of linear equations and inequalities is a more promising approach for demonstrating the advantages of parametric expressions. The simplest homogeneous systems are composed straight on the Petri net incidence matrix $C$ [20]:

$$
\bar{x} \cdot C=0
$$

to obtain the vector $\bar{x}$ of p-invariants and

$$
C \cdot \bar{y}=0
$$

to obtain the vector $\bar{y}$ of t-invariants. A net, having a $p$ invariant whose components are all positive, possesses an important property, i.e., boundedness. A positive $t$-invariant is a necessary condition for a net to be reversible, i.e., able to return to its initial marking from any reachable marking.

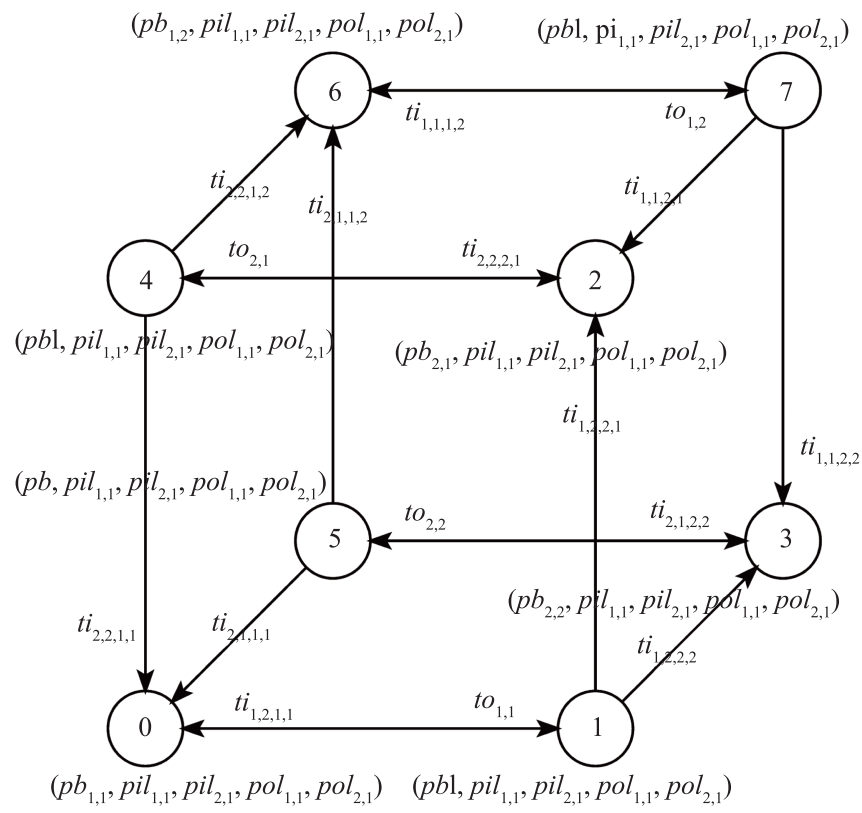

Fig. 3. Examples of Petri nets state space $(d=2$ and $k=1)$ for $p b_{1,1}^{1,1}=1$.

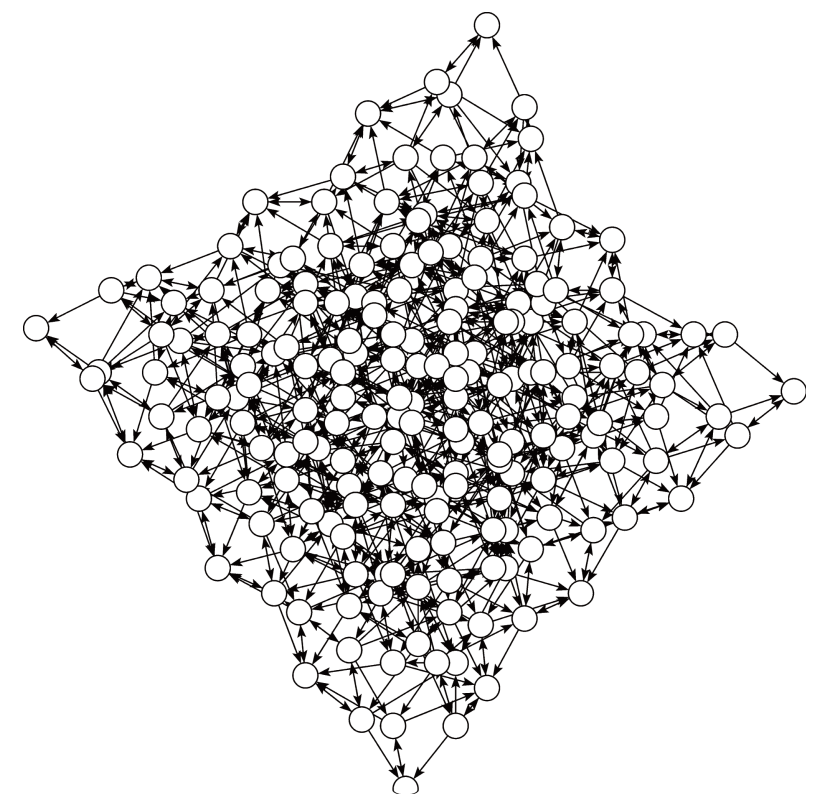

Fig. 4. Examples of Petri nets state space $(d=2$ and $k=1)$ for $p b_{j, n}^{1,1}=$ $1,1 \leq j \leq 2,1 \leq n \leq 2$.

Expression (2) describes a sparse matrix $C$ on rows. Thus, a system for computing $p$-invariants (specified in a parametric form) is directly obtained on the expression, and its parametric solution is sought. Had we found its parametric solution, we could infer the model properties for any given parameter that allows a conclusion for the properties of infinite Petri nets. For $H_{d, k}$ specified by (2)-(3), the system and its parametric 
solution are represented by (4) and (5), respectively.

$$
\left\{\begin{array}{c}
-x p b_{j, 1}^{\mathbf{i}}-x p o l_{j, 1}^{\mathbf{i}}+x p o_{j, 1}^{\mathbf{i}}+x p b l^{\mathbf{i}}=0 \\
-x p i_{j, 1}^{\mathbf{i}}-x p b l^{\mathbf{i}}+x p b_{j^{\prime}, n^{\prime}}^{\mathbf{i}}+x p i l_{j, 1}^{\mathbf{i}}=0 \\
-x p b_{j, 2}^{\mathbf{i}}-x p i l_{j, 1}^{x i(\mathbf{i}, j)}+x p i_{j, 1}^{x i(\mathbf{i}, j)}+x p b l^{\mathbf{i}}=0 \\
-x p o_{j, 1}^{x i(\mathbf{i}, j)}-x p b l^{\mathbf{i}}+x p b_{j^{\prime}, n^{\prime}}^{\mathbf{i}}+x p o l_{j, 1}^{x i(\mathbf{i}, j)}=0 \\
\mathbf{i}=\left(i_{1}, \ldots, i_{d}\right), 1 \leq i_{j} \leq k_{j}, \\
1 \leq j \leq d, \\
1 \leq j^{\prime} \leq d \\
1 \leq n^{\prime} \leq 2,\left(j^{\prime}, n^{\prime}\right) \neq(j, n) .
\end{array}\right.
$$

$$
\left(\begin{array}{c}
\left(p i_{j, 1}^{\mathbf{i}}, p i l_{j, 1}^{\mathbf{i}}\right): \mathbf{i}=\left(i_{1}, \ldots, i_{d}\right), 1 \leq i_{j} \leq k_{j}, 1 \leq j \leq d, \\
\left(p o_{j, 1}^{\mathbf{i}}, p o l_{j, 1}^{\mathbf{i}}\right): \mathbf{i}=\left(i_{1}, \ldots, i_{d}\right), 1 \leq i_{j} \leq k_{j}, 1 \leq j \leq d, \\
\left(\left\{p b_{j, n}^{\mathbf{i}}, p b l^{\mathbf{i}}\right\}: 1 \leq j \leq d, 1 \leq n \leq 2\right), \\
\mathbf{i}=\left(i_{1}, \ldots, i_{d}\right), 1 \leq i_{j} \leq k_{j}, \\
\left(\left\{p b l^{\mathbf{i}}, p i l_{j, 1}^{\mathbf{i}}, p o l_{j, 1}^{\mathbf{i}}\right\}:\right. \\
\left.\mathbf{i}=\left(i_{1}, \ldots, i_{d}\right), 1 \leq i_{j} \leq k_{j}, 1 \leq j \leq d\right) \\
\left(\left\{p b_{j, n}^{\mathbf{i}}, p i_{j, 1}^{\mathbf{i}}, p o_{j, 1}^{\mathbf{i}}\right\}:\right. \\
\left.\mathbf{i}=\left(i_{1}, \ldots, i_{d}\right), 1 \leq i_{j} \leq k_{j}, 1 \leq j \leq d, 1 \leq n \leq 2\right)
\end{array}\right)
$$

The parametric system (4) is directly constructed on expression (2). The prefix " $x$ " is added to denote the components of $p$-invariants. The specifics of system (4) follow from the fact that it is composed over a ring of integers. Moreover, its solutions are sought in a monoid of nonnegative integer numbers. Generally, there are no known methods to obtain a basic solution of (4). Using an ad-hoc technique on a series of models generated by the program htgen described in Section IV, we compose a parametric solution and then prove that it is a solution of the given system. For system (4), the composed solution is shown in (5).

Each of two first rows of (5) describes a few rows of a sparse matrix of basic solutions having only two nonzero (unitary) components. The third row represents a series of rows with a variable number of nonzero components. Each of the fourth and fifth row specifies a single row having a variable number (rather "big") of nonzero components. Note that for the 2dimensional case, (5) coincides with the expression obtained in [4], and it is accurate within the system of ports notations. For a plain case, a simpler clockwise enumeration of ports is applied in [4]. For a particular case when $d=2$ and $k=2$, the $p$-invariants obtained (generated) on (5) are listed in (6).

Note that there are a total of 22 vectors that coincide with the solutions calculated through Tina [11], [12].

Theorem 1: Equation (5) specifies solutions of (4).

Proof: We employ an exhaustive proof by substituting/inserting each row of the sparse matrix (5) into each parametric equation of (4). Here, we consider a case for the third row of (5):

$$
\left\{\begin{array}{l}
\left.\left(\begin{array}{l}
-x p b_{j_{1}}^{\mathbf{i}}+x p b l^{\mathbf{i}}=0 \\
-x p b l^{\mathbf{i}}+x p b_{j^{\prime}, n^{\prime}}^{\mathbf{i}}=0 \\
-x p b_{j_{2}}^{\mathbf{i}}+x p b l^{\mathbf{i}}=0 \\
-x p b l^{\mathbf{i}}+x p b_{j^{\prime}, n^{\prime}}^{\mathbf{i}}=0
\end{array}\right): \begin{array}{l}
\mathbf{i}=\left(i_{1}, \ldots, i_{d}\right), 1 \leq i_{j} \leq k_{j}, \\
1 \leq j \leq d, \\
1 \leq j^{\prime} \leq d, \\
\end{array}\right),\left(j^{\prime}, n^{\prime}\right) \neq(j, n) .
\end{array}\right.
$$

Because the third row of (5) contains nonnegative $x p b_{j, n}^{\mathbf{i}}$ for all combinations of internal parameters $j$ and $n$, we obtain
$-1+1=0$ in each equation. We proceed similarly with the rest of the vectors.

$$
\begin{aligned}
& \left(p i_{1,1}^{1,1}, p i l_{1,1}^{1,1}\right),\left(p i_{1,1}^{1,2}, p i l_{1,1}^{1,2}\right) \\
& \left(p i_{1,1}^{2,1}, p i l_{1,1}^{2,1}\right),\left(p i_{1,1}^{2,2}, p i l_{1,1}^{2,2}\right) \\
& \left(p i_{2,1}^{1,1}, p i l_{2,1}^{1,1}\right),\left(p i_{2,1}^{1,2}, p i l_{2,1}^{1,2}\right) \\
& \left(p i_{2,1}^{2,1}, p i i_{2,1}^{2,1}\right),\left(p i_{2,1}^{2,2}, p i l_{2,1}^{2,2}\right) \\
& \left(p_{1,1}^{1,1}, p_{1,1}^{1,1}\right),\left(p o s_{1,1}^{1,2}, p_{1,1}^{1,2}\right) \\
& \left(p o_{1,1}^{2,1}, p o l_{1,1}^{2,1}\right),\left(p o_{1,1}^{2,2}, p o l_{1,1}^{2,2}\right) \\
& \left(p o_{2,1}^{1,1}, p_{2} l_{2,1}^{1,1}\right),\left(p o_{2,1}^{1,2}, p_{2}^{1,2} l_{2,1}^{1,2}\right) \\
& \left(p o o_{2,1}^{2,1}, p o l_{2,1}^{2,1}\right),\left(p o o_{2,1}^{2,2}, p o l_{2,1}^{2,2}\right) \\
& \left(p b_{1,1}^{1,1}, p b_{1,2}^{1,1}, p b_{2,1}^{1,1}, p b_{2,2}^{1,1}, p b l^{1,1}\right) \\
& \left(p b_{1,1}^{1,2}, p b_{1,2}^{1,2}, p b_{2,1}^{1,2}, p b_{2,2}^{1,2}, p b l^{1,2}\right) \\
& \left(p b_{1,1}^{2,1}, p b_{1,2}^{2,1}, p b_{2,1}^{2,1}, p b_{2,2}^{2,1}, p b l^{2,1}\right) \\
& \left(p b_{1,1}^{2,2}, p b_{1,2}^{2,2}, p b_{2,1}^{2,2}, p b_{2,2}^{2,2}, p b l^{2,2}\right) \\
& \left(p b l^{1,1}, p b l^{1,2}, p b l^{2,1}, p b l^{2,1}, p i l_{1,1}^{1,1}, p i l_{1,1}^{1,2}\right. \text {, } \\
& p i l_{1,1}^{2,1}, p i l_{1,1}^{2,2}, p i l_{2,1}^{1,1}, p i l_{2,1}^{1,2}, p i l_{2,1}^{2,1}, p i l_{2,1}^{2,2} \text {, } \\
& \left.\operatorname{pol}_{1,1}^{1,1}, \operatorname{pol}_{1,1}^{1,2}, \text { pol }_{1,1}^{2,1}, \text { pol }_{1,1}^{2,2}, \text { pol }_{2,1}^{1,1}, \text { pol }_{2,1}^{1,2}, \text { pol }_{2,1}^{2,1}, \text { pol }_{2,1}^{2,2}\right) \text {, } \\
& \left(p b_{1,1}^{1,1}, p b_{1,1}^{1,2}, p b_{1,1}^{2,1}, p b_{1,1}^{2,2}, p b_{1,2}^{1,1}, p b_{1,2}^{1,2}, p b_{1,2}^{2,1}, p b_{1,2}^{2,2},\right. \\
& p b_{2,1}^{1,1}, p b_{2,1}^{1,2}, p b_{2,1}^{2,1}, p b_{2,1}^{2,2}, p b_{2,2}^{1,1}, p b_{2,2}^{1,2}, p b_{2,2}^{2,1}, p b_{2,2}^{2,2} \text {, } \\
& p i_{1,1}^{1,1} p i_{1,1}^{1,2}, p i_{1,1}^{2,1}, p i_{1,2}^{2,2}, p i_{2,1}^{1,1}, p i_{2,2}^{1,2}, p i_{2,1}^{2,1}, p i_{2,2}^{2,2} \\
& p i_{1,1}^{1,}, p i_{1,2}^{1,1}, p i_{1,1}^{2,1}, p i_{1,1}^{2,2}, p i_{2,1}^{1,1}, p i_{2,1}^{1,2}, p i_{2,1}^{2,}, p i_{2,1}^{2,2} \\
& \left.p o_{1,1}^{1,1}, p o_{1,1}^{1,2}, p o_{1,1}^{2,1}, p o_{1,1}^{2,2}, p o_{2,1}^{1,1}, p o_{2,1}^{1,2}, p o_{2,1}^{2,1}, p o_{2,1}^{2,2}\right)
\end{aligned}
$$

Theorem 2: $H_{d, k}$ is a bounded and conservative Petri net for any number of dimensions $d$, and sizes $k$.

To prove Theorem 2, we do not need a matrix of basic solutions for (4). We compose an invariant, namely, the sum of the fourth and fifth rows of (5), and have all components equal to the unit. To double check the technique, we note that $H_{d, k}$ is a priori bounded and safe because each of its transitions has the same number of incoming and outgoing arcs.

In the same way, using dual parametric expressions [1], a parametric system for computing $t$-invariants is composed. In a majority of studied cases, for rather small instances of Petri nets, an explosion of basic solutions is observed that hampers their parametric description. To deal with $t$-invariants and observe deadlocks for infinite Petri nets, auxiliary graphs are introduced and studied in [5].

T-invariance is a necessary condition of liveness of bounded nets. A deadlock, which occurs when no transition can fire, represents a negative property. A method to find and overcome deadlocks, called "liveness-enforcing," is studied in [21], [25]-[29]. The technique is based on solving systems of equations and inequalities that can be easily composed in a parametric form based on the parametric representation of the incidence matrix.

\section{Program to Generate Hypertorus Petri Nets}

As mentioned in Section III, basic methods of analyzing infinite Petri nets [1], [2] consist of solving linear systems of equations (inequalities) for finding place and transition invariants to prove properties of an ideal protocol. At present time, universal methods of solving infinite (parametric) Diophantine systems in nonnegative integer numbers are unknown. That is why our inductive ad-hoc approach is based on generating 
a sequence of models of growing size, finding invariants for each model, and finally abstracting for generalizing a sequence of solutions in a parametric form. After this not completely formalized process, we prove that the obtained parametric matrix represents a basic solution of the infinite system as shown in Section III. To automate the process of composing models for different values of parameters, we need a program that allows us to avoid manual creation of models. Below, we describe such a program called htgen and its usage.

\section{A. Description of Use}

The program htgen generates a hypertorus Petri net model in the textual .net format of Tina [21] with a given number of dimensions $d$ and the same size $k$ on all dimensions. Additional parameters specify the initial marking. The program is written in the language ANSI C and compiled with the following command:

\section{$>$ gcc - o htgen htgen.c}

The program htgen is run on the command line using the following command:

$>$ htgen d k p b > ht_model.net

Here $d$ specifies the number of dimensions, $k$ specifies the hypertorus size in each dimension, $p$ specifies the number of packets in each internal buffer of each cell, and $b$ specifies the available size of the internal buffer for each cell. The resulting file $h t$ model.net is processed either in the integrated environment $n d$ of Tina or using its separate modules such as struct, tina, and sift or plug-ins of Tina such as Deborah and Adriana. It could be exported as well in a few other formats common for the exchange of Petri net models.

\section{B. The Output Format and the Principles of Operation}

The textual format of time Petri nets in Tina [11], [12] specifies a Petri net with lines starting with the keyword "tr" $\operatorname{tr}\{\mathrm{t}$-name $\}\{\mathrm{p}$-name $\}\left[{ }^{\prime} *\right.$ ' $<$ weight $\left.>\right], \ldots->$

$\{$ p-name $\}[$ '*' $<$ weight $>], \ldots$

and the initial marking of the Petri net is specified with lines starting with "pl":

pl $\{$ p-name $\}(<$ marking $>)$

The fields in the lines starting with "tr" and "pl" directly correspond to the structure of the parametric expressions (2) and (3), respectively.

The operation of the program htgen consists of generating all the lines of (2) and (3) according to the index ranges given in the input parameters. Each place $(p-)$ and transition $(t-)$ includes the upper index in the $d$-dimensional grid and the lower index either of a port (surface) for output tracts or a pair of ports for an input tract. The port index is specified with pair $(j, n)$, where the number $j$ defines the dimension and the number $n$ defines the direction ( 1 for the origin and 2 for infinity).

While the port index represents a pair, the grid cell index is a collection having $d$ components. We use the macros PUTINDX and PUTINDXNEXT. The macro PUTINDX generates a variable length index of the current grid cell and the macro PUTINDXNEXT generates a variable length index of the next grid cell. The indices of a cell are printed sequentially after the cell with a dot separator, where first the lower index (indices) of a port (a pair of ports) and then the upper index of the grid cell are printed. The character ' $d$ ' is printed after the number indicating the dimension and the character ' $\mathrm{n}$ ' is printed after the number for the direction. For instance "ti.d1.n1.d1.n2.1.3" corresponds to $t i_{1,1,1,2}^{1,3}$.

\section{The Description of the Data Structures and Macros}

To generate a hypertorus with a given dimension $d$, the cell index $i$ is dynamically allocated

$\mathrm{i}=($ int $*) \operatorname{malloc}((\mathrm{d}+1) * \operatorname{sizeof}(\mathrm{int}))$;

We start indexing directly from one but we allocate an extra index for zero which remains unused. To print the index of a current cell we use the following macro:

\#define PUTINDX $\{$ for $(\mathrm{u}=1 ; \mathrm{u}<=\mathrm{d} ; \mathrm{u}++$ ) printf(".\%d", i[u] );

The indices of each dimension are printed using a dot character as a separator. To print the index of the next cell we use the following macro:

\#define PUTINDXNEXT( $\mathrm{r})\{$ for $(\mathrm{u}=1 ; \mathrm{u}<=\mathrm{d}$; $\mathrm{u}++$ ) printf(".\%d", $(\mathrm{u} !=(\mathrm{r}))$ ? i $[\mathrm{u}]$ : $(\mathrm{i}[\mathrm{u}]<\mathrm{k})$ ? $\mathrm{i}[\mathrm{u}]+1: 1) ;\}$

This macro encodes the torus composition rule: the next cell index is incremented if $i[u]<k$, and it is connected to the first cell if $i[u]=k$.

\section{The Description of the Algorithm}

The algorithm encodes expressions (2)-(3) which avoids the dependence of the variable in the loop on the actual number of dimensions when using $d$ nested loops to enumerate all cells. Instead we consider the cell index as a $d$ digit number in a radix system with radix $k+1$ without zero digits. We initialize the index $i$ with the smallest value corresponding to all components equal to one:

for $(\mathrm{j}=1 ; \mathrm{j}<=\mathrm{d} ; \mathrm{j}++) \mathrm{i}[\mathrm{j}]=1$;

At each pass of the main loop

while( loop ) $\{\ldots\}$

we print the model of the current cell and then calculate the next cell index by adding one to the lowest digit of the index and moving the carry to the left. In case this is impossible, when the maximal index having all components equal to $k$ has been processed, the loop indicator loop is reset and the main loop is abandoned:

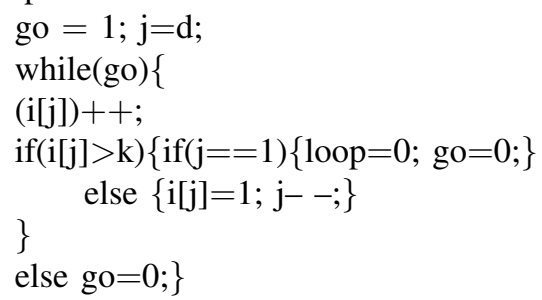

The content of the model of the current cell is classified on elements bound to a facet and bound to the cell; there is only one vertex where the internal buffer size does not depend on the facet index, and it is generated as the last element as follows.

printf(“pl \{pbl”);

PUTINDX; 
printf(“\} (\%d)\n”, b);

To enumerate all the ports of the current cell we use a loop over the dimension $j$

for $(\mathrm{j}=1 ; \mathrm{j}<=\mathrm{d} ; \mathrm{j}++)\{\ldots\}$

and list explicitly two facets for directions 1 and 2 .

At first, we generate lines for direction 1 using the macro PUTINDX because the indices of the ports in the direction of the origin coincide with the indices of the current cell. For the output tract, a transition "to. $<\mathrm{j}>\mathrm{d} .1 \mathrm{n}$.*" is generated. For the input tract, we use a double loop on the dimension $v$ and the direction $d i r$ of the destination port, respectively; $2 \cdot d-1$ transitions "ti. $<\mathrm{j}>$ d. $1 \mathrm{n} .<\mathrm{v}>$ d. $<$ dir $>$ n. $*$ " are generated.

Then we proceed with lines for direction 2 in the same manner but use the macro PUTINDXNEXT for contact places because they contain the next cell index. Note that, an input tract maps into the corresponding output tract of the next cell and vice versa.

\section{E. Some Examples}

The following command line:

$>$ htgen $2332>$ ht2d3k3p2b.net

generates a hypertorus model of size 3 in a 2-dimensional space; each cell has 3 packets in each of its buffer section and the available size of the buffer equals 2 .

Then we start module nd of the Tina toolbox [21] and load the model. To visualize the model, we choose the following sequence in the menu: "Edit-draw-neato". The result is shown in Fig. 5 for the 2-dimentional torus. Note that automatic visualization of the multidimensional models reflects the traces of a general structure and can be edited manually.

To watch the behavior of the model where we see tokens moving around, we choose the menu sequence: "Tools-stepper simulator-Rand".

Moreover, within the Tina toolbox we can construct a state space of the model by using "reachability analysis" and we can calculate $p$-and $t$-invariants by using the tool for "structural analysis".

For the analysis of a big model Tina offers a series of separate modules, for instance, the tool sift constructs a state space rather fast and the tool muse allows to query for the properties of the constructed state space.

To use other tools for Petri nets analysis, Tina exports nets in a few standard Petri net formats.

\section{MOdeling HyPERTORUS GRIDS BY PROCESS ALGEBRA}

A motivation for modeling hypertorus grids with process algebra is the availability of efficient tools for protocols analysis. As it is shown in Section VI, modeling hypertorus grids in mCRL2 [14]-[16] brings better performance than applying one of the best tools for Petri nets-Tina [10], [12]. While we prove $p$-and $t$-invariance for any values of the grid size and dimension using the abstraction of infinite Petri nets, there is no known technique to prove liveness and the presence/absence of deadlocks. Using mCRL2 modelchecking we can prove these properties for concrete values of parameters.

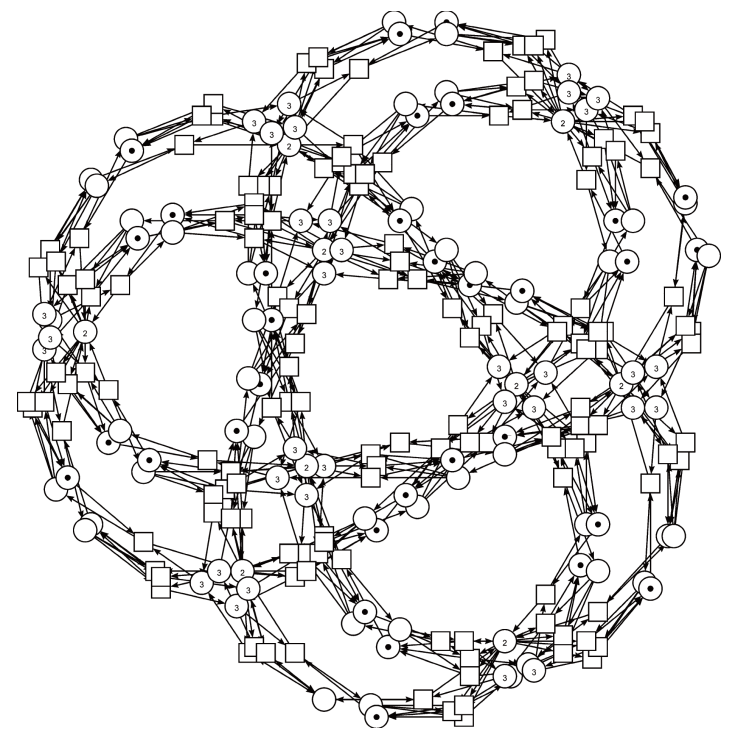

Fig. 5. Automatic visualization of the generated models in Tina of a 2 dimensional torus (ht2d3k3p2b.net).

mCRL2 [14], [15] is a formal specification language with an associated toolset which is based on the Algebra of Communicating Processes (ACP) extended to include data and time. The toolset can be used for modelling, validation and verification of concurrent systems and protocols. The toolset supports a collection of tools for linearization, simulation, state-space exploration and generation, and tools to optimize and analyze specifications. Moreover, state spaces can be manipulated, visualized and analyzed. A model consists of three basic parts for specification of actions (act), processes (proc), and initialization (init).

Let us consider a model of a $3 \times 3$ torus in a 2-dimensional space for a grid without buffers of ports. This is represented with the following listing:

act

in 1 , out 1 , in 2 , out 2 , in 3 , out 3 , in 4 , out 4 ,

c13, c24, c31, c42: Nat\#Nat;

map GridSize: Nat;

eqn GridSize $=3$;

map prev, next: Nat->Nat;

var n: Nat;

eqn $\operatorname{prev}(\mathrm{n})=\mathrm{if}(\mathrm{n}==1$, GridSize, $\max (0, \mathrm{n}-1))$;

$\operatorname{next}(\mathrm{n})=\operatorname{if}(\mathrm{n}==$ GridSize, $1, \mathrm{n}+1)$;

proc

$\mathrm{d}(\mathrm{b} 1, \mathrm{~b} 2, \mathrm{~b} 3, \mathrm{~b} 4, \mathrm{bl}:$ Int, i, j: Nat) $=$

$\%$ output

((b1>0)->out1(prev(i), j).d(b1-1, b2, b3, b4, bl+1, i, j))+ $((b 2>0)->$ out2(i, prev(j)).d(b1, b2-1, b3, b4, bl+1, i, j)) + $((b 3>0)->$ out3(next(i), j).d(b1, b2, b3-1, b4, bl+1, i, j)) + ((b4>0)->out4(i, next(j)).d(b1, b2, b3, b4-1, bl+1, i, j))+ $\%$ input \& switching (nondeterministic)

$((\mathrm{bl}>0)->($

$\%$ from port 1

in $1(i, j) \cdot d(b 1, b 2+1, b 3, b 4, b 1-1, i, j)+$ in1(i, j).d(b1, b2, b3+1, b4, bl-1, i, j)+ in1(i, j).d(b1, b2, b3, b4+1, bl-1, i, j)+ 


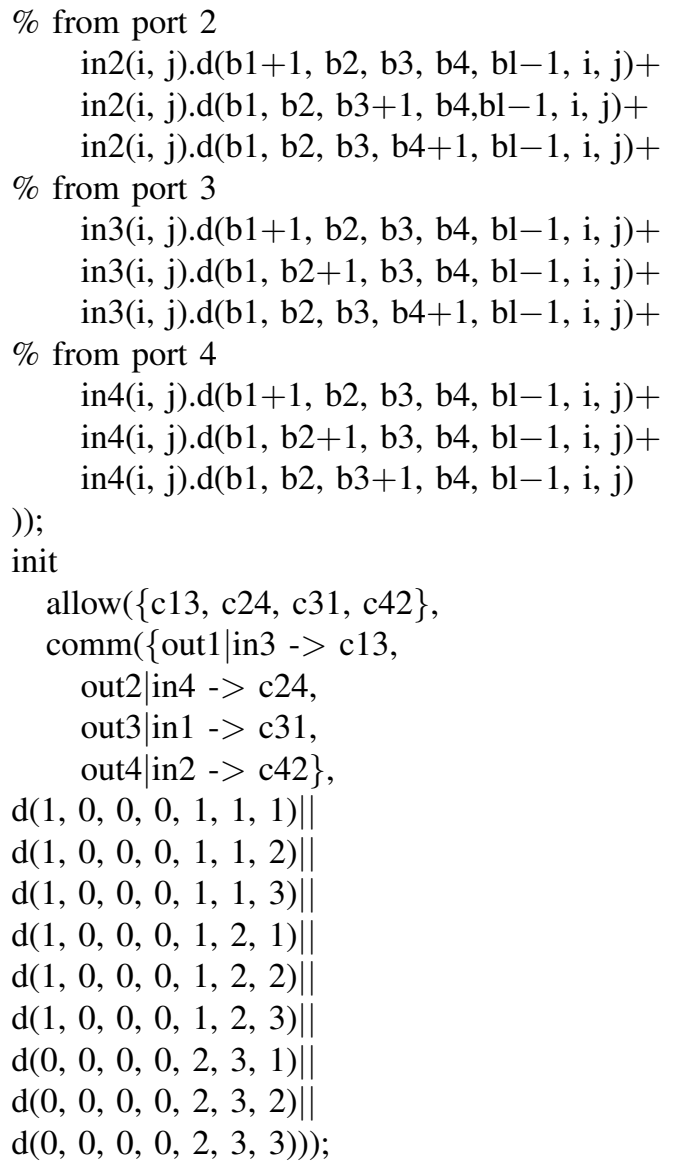

Basic actions are the input and output of a packet via one of the four ports, represented with in 1 , out1, in2, out 2 , in 3 , out 3 , in 4 , out 4 and the communications between adjacent ports of neighboring devices represented with c13, c24, c31, c42; note that the mentioned actions are indexed on the grid with a pair of natural indexes Nat\#Nat. A grid size is given as the mapping called GridSize. Then two functions prev and next are specified which return the number of the previous and the next node, respectively taking into consideration the fact that the opposite borders are closed.

As a basic process, a grid node $d$ is specified describing its output and input separately; the parameters b1, b2, b3, b4 and bl specify sections of the internal buffer and its current size, respectively while variables $\mathrm{i}$ and $\mathrm{j}$ give the node location within the grid. When a packed is output via port $\mathrm{k}$, the node issues the corresponding action out $k$, which decrements the corresponding buffers section size $\mathrm{b}_{k}$, and increments the buffer length bl. The packet input is more sophisticated because it is combined with a nondeterministic forwarding decision, and the corresponding action for input from port $\mathrm{k}$ is represented $\mathrm{k}$ is an index.

The initialization section with $\operatorname{in}_{k}(i, j)$ specifies the allowed actions and the communication between neighboring nodes; for instance, the notation "out1|in3 - > c13" means that output via port 1 and input via port 3 of the neighboring node results in the communication c13. Note that the indices of actions are calculated by the functions prev and next. Then an explicit parallel composition of all participating processes follows. The last pair of indices specifies the position of the process in the grid while the first 5 indices specify the initial state of the output buffers of each grid process.

The model has been analysed with the mCRL2 tools. It functions completely in accordance with the behavior of the corresponding Petri net model [30] shown in Fig. 6.

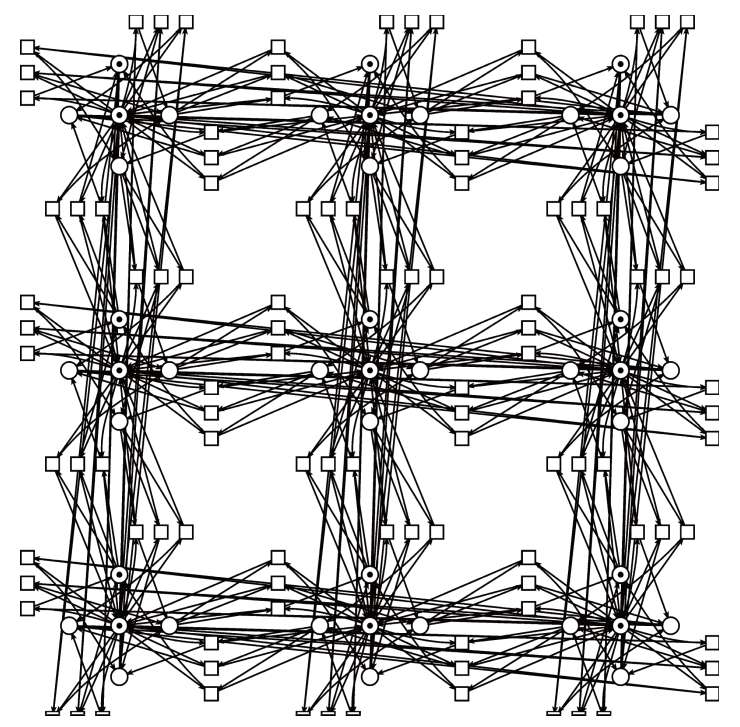

Fig. 6. Model of the $3 \times 3$ grid in 2-dimensional space without ports' buffers.

In the same way as described in Section IV, a generator ht-mcrl2-gen of hypertorus grid models in mCRL2 has been developed [13]; it is supplied with a generator of the corresponding Petri net model as well.

Mutual automatic transformations of a Petri net and mCRL2 models represent a future work direction.

\section{BENCHMARKS FOR StATE SPACE ANALYSIS AND FINDING DEADLOCKS WITH TINA AND MCRL2}

Thorough analysis of the benchmarks results revealed that it is useful to employ both Petri nets and process algebra analysis techniques. Two systems have been chosen as representatives of the corresponding classes: Tina for Petri nets as a winner of the Model-Checking Contest [31] and mCRL2 [14], [15] for process algebra. We use simplified models without buffers of ports as studied in Section V.

Three tests have been implemented on series of models with increasing dimension and size:

T1) The construction of a complete state space;

T2) A state space query - to check liveness and check boundedness;

T3) "on-the-fly" search of the first deadlock.

Note that mCRL2 implements a very powerful highway search [16] technique. We use a Dell Inspiron 15, Series 500 laptop with an Intel Core i5-5200U $2.2 \mathrm{GHz}$ processor (2 cores) and 8 Gigabytes of RAM. The required script commands are represented in Table I. The obtained benchmarks shown in Fig. 7 acknowledge the considerable advantages of mCRL2. 
TABLE I

COMMAND LINES FOR TESTS

\begin{tabular}{|c|c|c|}
\hline Test & System & Command lines \\
\hline \multirow{2}{*}{$\mathrm{T} 1$} & Tina & $>$ sift $-\mathrm{R} \$ 1 . \mathrm{ndr}$ \\
\hline & mCRL2 & $>$ mcrl22lps -v \$1.merl2 \$1.lps \\
\hline \multirow{5}{*}{$\mathrm{T} 2$} & Tina & $>$ selt $\$ 1 . k t z \$ 2.1 t 1$ \\
\hline & \multirow{4}{*}{ mCRL2 } & $>$ lps2pbes \$1.lps -c -f \$2.mcf \$1_\$1.pbes \\
\hline & & $>$ pbessolve $-\mathrm{v}$ \\
\hline & & -evidence-file $=\$ 1 \_\$ 2 \_c o u n t e r \_$example.lps $-\mathrm{f} \$ 1.1 p s$ \\
\hline & & -s2 -rjitty $\$ 1 \_\$ 2 . p b e s$ \\
\hline \multirow{2}{*}{$\mathrm{T} 3$} & Tina & $>$ sift $-\mathrm{R}$-df -dead \$1.ndr \\
\hline & mCRL2 & $>$ lps21ts -D -vrjittyc -todo-max $=2 \$ 1.1 p s-t 1$ \\
\hline
\end{tabular}

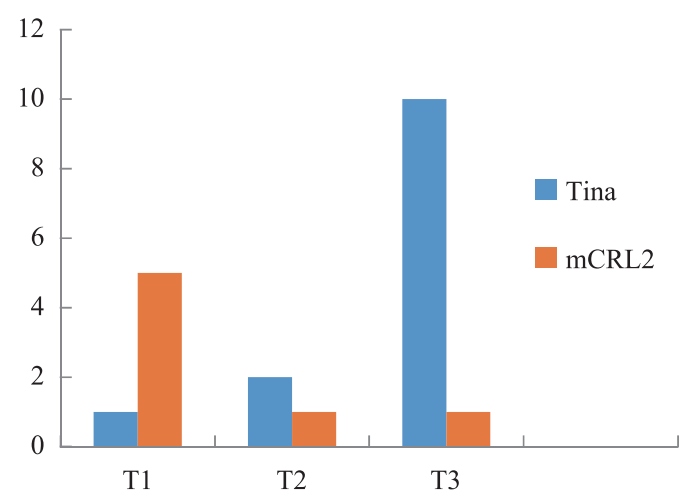

Fig. 7. Benchmarks (in times of slow-down).

Within the command lines the parameter indicated by " $\$ 1$ " specifies the model file name, while the parameter indicated by " $\$ 2$ " specifies a file containing a query on the state space of the model. The state space of a Petri net is represented by its reachability graph [20] and stored as a Kripke transition system in binary format ".ktz"; queries are written in the linear time temporal logic notation (LTL) in a file with extension ".lt1".

For mCRL2 we have the following file formats: a linear process specification ".lps" that is a symbolic representations of a state space for mCRL2 models; a labelled transition system ".lts" is used for visualization and export; a modal $\mu$-calculus formula ".mcf" specifies queries; parameterized Boolean equation systems ".pbes" specifies the model state space integrated with a query. Note that mCRL2 offers a nice 3D utility ltsview for state space visualization; an example of the state space visualization of ht $2 \mathrm{~d} 2 \mathrm{k} 1 \mathrm{p} 2 \mathrm{~b} . \mathrm{mcr} 2 \mathrm{is}$ shown in Fig. 8; a "helmet" with "belts" allows us to observe deadlocks as "bids". Moreover, when a negative answer is obtained, the first counter-example is stored and can be visualized as well in $2 \mathrm{D}$ visualization program ltsgraph.

The queries to check liveness for Tina are formulated in LTL as follows:

$$
\begin{aligned}
& {[]<>\{\text { ti_1,1^1,1\}/ }} \\
& {[]<>\left\{\text { ti_l, } 1,2^{\wedge} 1,1\right\} \wedge} \\
& \text {... } \\
& \text { [] }<>\{\text { ti_2,2^2,2\}; }
\end{aligned}
$$

The queries in mCRL2 have the following form as modal $\mu$-calculus formulas: ([true*]forall $\quad \mathrm{x}, \mathrm{y}: \operatorname{Nat} . v a l(\mathrm{x}<=2 \quad \& \& \quad \mathrm{y}<=2) \quad=>$ $<$ true*.exists d:Direction.c1n1t1n2(x,y,d) $>$ true $) \& \&$

([true*]forall $\quad \mathrm{x}, \mathrm{y}: \operatorname{Nat} . v a l(\mathrm{x}<=2 \quad \& \& \quad \mathrm{y}<=2) \quad=>$ $<$ true*.exists d:Direction.c1n2t $1 \mathrm{n} 1(\mathrm{x}, \mathrm{y}, \mathrm{d})>$ true $) \& \&$

([true*]forall $\mathrm{x}, \mathrm{y}: \operatorname{Nat} . v a l(\mathrm{x}<=2 \quad \& \& \quad \mathrm{y}<=2) \quad=>$ $<$ true*.exists d:Direction.c2n1t2n2(x,y,d $>$ true $) \& \&$

([true*]forall $\quad \mathrm{x}, \mathrm{y}: \operatorname{Nat} . v a l(\mathrm{x}<=2 \quad \& \& \quad \mathrm{y}<=2) \quad=>$ $<$ true*.exists d:Direction.c2n2t2n1(x,y,d) $>$ true $) \& \&$ true

The benchmarks show that mCRL2 takes more time to build the state space. We consider a state space as a labelled transition system which is used for exchange with other systems, and when building an ".lps" file, which is used to generate systems of equations to evaluate queries, it runs about hundred times faster than Tina. When implementing state space queries, and especially when finding the first deadlock, mCRL2 also runs considerably faster.

Thus, we recommend using both approaches because of their mutually complementing nature.

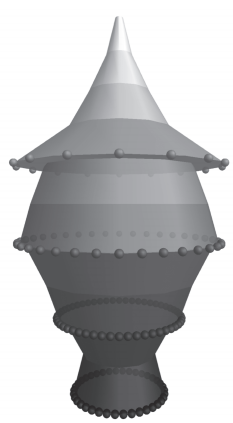

Fig. 8. Visualization of ht2d2k1p2b.mcrl2 state space with ltsview.

\section{CONCLUSION}

A hypertorus communication grid is specified by an infinite Petri net. Inductive composition of $\mathrm{p}$ - and t-invariants gives a uniform solution in a parametric form to draw conclusions on infinite nets such as boundedness, conservativeness and consistency for any number of dimensions and any size of grid. Some remarks regarding liveness and liveness enforcing are presented.

The developed programs htgen and ht-mcrl2-gen automatically generate Petri net and process algebra models with a hypertorus grid structure. The models have been analyzed with Tina and mCRL2, and the obtained benchmarks have been compared. The results show certain benefits of mCRL2 for finding deadlocks while Tina is faster for state space queries. This justifies the application of both approaches.

Large-size Petri net and process algebra models with known properties provide a testset for model-checking software. Applications in systems biology, artificial intelligence, and thermonuclear physics are foreseen and should be sought.

\section{ACKNOWLEDGMENT}

The authors thank the Fulbright Program and Prof. MengChu Zhou for the opportunity to present infinite Petri nets at a dedicated seminar in NJIT on October 11, 2017, by the first author. 


\section{REFERENCES}

[1] D. A. Zaitsev, I. D. Zaitsev, and T. R. Shmeleva, "Infinite petri nets: part 1, modeling square grid structures," Complex Systems, vol. 26, no. 2, pp. 157-195, 2017.

[2] D. A. Zaitsev, I. D. Zaitsev and T. R. Shmeleva, "Infinite petri nets: part 2, modeling square grid structures," Complex Systems, vol. 26, no. 4, pp. $341-371,2017$.

[3] D. A. Zaitsev and T. R. Shmeleva, "Verification of hypercube communication structures via parametric Petri nets," Cybernetics and Systems Analysis, vol. 46, no. 1, pp. 105-114, 2010

[4] D. A. Zaitsev, "Verification of computing grids with special edge conditions by infinite petri nets," Automatic Control and Computer Sciences, vol. 47, no. 7, pp. 403-412, 2013.

[5] T. R. Shmeleva, D. A. Zaitsev, and I. D. Zaitsev, "Verification of square communication grid protocols via infinite Petri nets," in Proc. of MESM 10th Middle Eastern Simulation Multiconference, 2009, 53-59.

[6] S. Cheng, W. Zhong, and K. E. Isaacs, and K. Mueller, "Visualizing the topology and data traffic of multi-dimensional torus interconnect networks," IEEE Access, 2018.

[7] N. P. Preve (Ed.), Grid Computing: Towards a Global Interconnected Infrastructure, Springer, 2011.

[8] D. Gilbert, M. Heiner, F. Liu, and N. Saunders, "Colouring space - a coloured framework for spatial modelling in systems biology," in Proc. Conf. Petri Nets, Milano, Springer, LNCS, vol. 7927, pp. 230-249, June 2013.

[9] K. Miyamoto, Plasma Physics and Controlled Nuclear Fusion. Springer, 2005.

[10] L. V. Belevtsov and A. A. Kostikov, "Influence of grain properties on the Abrikosov vortices interaction with Josephson junction," Journal of Low Temperature Physics, vol. 139, no. 1, pp. 11-19, 2005.

[11] B. Berthomieu, O.-P. Ribet, and F. Vernadat, "The tool TINAconstruction of abstract state space for Petri nets and time Petri nets," Int. J. Prod. Res., vol. 42, no. 14, pp. 2741-2756, 2004.

[12] B. Berthomieu et al., "Tina: Time Petri Net Analyser," LAAS, 2019 [Online]. Available: http:/projects.laas/fr/tina

[13] D. A. Zaitsev, "Software of Dmitry Zaitsev," GitHub, 2019 [Online]. Available: https://github.com/dazeorgacm/

[14] J. F. Groote and M. R. Mousavi. Modeling and Analysis of Communicating Systems. The MIT Press, 2014.

[15] J. F. Groote et al, "mCRL2: Analysing System Behaviour," TU/e, 2019. [Online]. Available: https://www.mcrl2.org/

[16] T. A. N. Engels, J. F. Groote, M. J. van Weerdenburg, and T. A. C. Willemse, "Search algorithms for automated validation," Journal of Logic and Algebraic Programming, vol. 78, no. 4, pp. 274-287, 2009.

[17] D. A. Zaitsev, "A generalized neighborhood for cellular automata," Theoretical Computer Science, vol. 666, pp. 21-35, March 2017.

[18] G. Berthelot and R. Terrat, "Petri nets theory for the correctness of protocols," IEEE Trans. on Communications, vol. 30, no. 12, pp. 2497$2505,1982$.

[19] M. Diaz, "Modelling and analysis of communication and cooperation protocols using petri net based model," Computer Networks, no. 6. pp. 419-441, 1982.

[20] T. Murata, "Petri Nets: Properties, Analysis and Applications," Proc. of the IEEE, vol. 77, no. 4, pp. 541-580, 1989.
[21] Z. W. Li and M. C. Zhou, Deadlock Resolution in Automated Manufacturing Systems, Springer, 2010.

[22] D. A. Zaitsev, "Decomposition of petri nets," Cybernetics and Systems Analysis, vol. 40, no. 5, pp. 739-746, 2004.

[23] J. Ye, M. C. Zhou, Z. Li, and A. Al-Ahmari, "Structural Decomposition and Decentralized Control of Petri Nets," IEEE Transactions on Systems, Man, and Cybernetics: Systems, pp. 1-10, DOI: 10.1109/TSMC.2017.2703950, July 2017.

[24] D. A. Zaitsev and A. I. Sleptsov, "State Equations and Equivalent Transformation for Timed Petri Nets," Cybernetics and Systems Analysis, vol. 33, no. 5, pp. 659-672, 1997.

[25] J. Luo, H. Ni, W. Wu, S. Wang, and M. C. Zhou, "Simultaneous reduction of petri nets and linear constraints for efficient supervisor synthesis," IEEE Trans. on Automatic Control, vol. 60, no. 1, pp. 88103, Jan. 2015.

[26] N. Wu, M. C. Zhou, and G. Hu, "Petri net modeling and one-step lookahead maximally permissive deadlock control of automated manufacturing systems," ACM Transactions on Embedded Computing Systems, vol. 12, no. 1, pp. 10:1-10:23, Jan. 2013

[27] H. Liu, K. Xing, W. Wu, M. C. Zhou, and H. Zou, "Deadlock prevention for flexible manufacturing systems via controllable siphon basis of petri nets," IEEE Trans. Systems, Man, and Cybernetics: Systems, vol. 45, no. 3, pp. 519-529, Mar. 2015.

[28] D. You, S. Wang, and M. C. Zhou, "Synthesis of monitor-based livenessenforcing supervisors for S3PR with $\xi$-resources," IEEE Trans. Systems, Man, and Cybernetics: Systems, vol. 45, no. 6, pp. 967-975, June 2015.

[29] H. Chen, N. Wu, and M. C. Zhou, "A novel method for deadlock prevention of AMS by using resource-oriented petri nets," Information Sciences, vol. 363, pp. 178-189, Oct. 2016.

[30] D. A. Zaitsev, T. R. Shmeleva, W. Retschitzegger, and B. Proll, "Security of grid structures under disguised traffic attacks," Cluster Computing, vol. 19, no. 3, pp. 1183-1200, 2016.

[31] F. Kordon et al., "Model Checking Contest," Sorbonne, 2019 [Online]. Available: https://mcc.lip6.fr

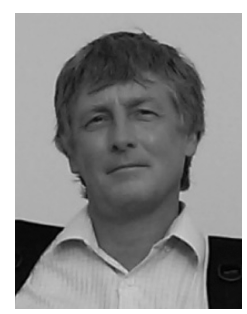

Dmitry A. Zaitsev (M'10-SM'11) received an Eng. degree in applied mathematics from Donetsk Polytechnic Institute, Donetsk, Ukraine, in 1986, a Ph.D. degree in automated control from the Kiev Institute of Cybernetics, Kiev, Ukraine in 1991, and a Dr.Sc. degree in telecommunications from the Odessa National Academy of Telecommunications, Odessa, Ukraine, in 2006.

$\mathrm{He}$ is a Professor of Computer Engineering at the International Humanitarian University, Odessa, Ukraine since 2009 and also a Professor of Computer Science at Vistula University, Warsaw, Poland since 2014. He developed the analysis of infinite Petri nets with regular structure, the decomposition of Petri nets in clans, and a method of synthesis of fuzzy logic functions given by tables. His current research interests include Petri net theory and its application in networking, production control, and computing. Recently he has been a co-director in Austria-Ukraine, China-Ukraine, and Slovakia-Ukraine research projects. He developed small universal Petri nets, the analysis of infinite Petri nets with a regular structure, the decomposition of Petri nets in clans, and the method of synthesis of fuzzy logic function given by tables. $\mathrm{He}$ designed the Opera-Topaz system for production control, models of protocols and networking technologies such as TCP, BGP, IOTP, MPLS, Bluetooth and PBB. Finally, he provided an implementation of the networking protocols E6 for the Linux kernel. He is a Senior Member of ACM. 


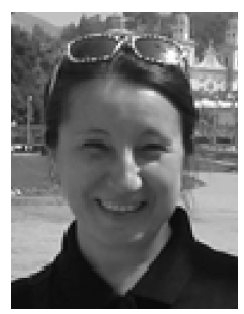

Tatiana R. Shmeleva received an Eng. degree in applied mathematics from the Moscow Railroad Engineering Institute in 1990, a Ph.D. degree in telecommunications from the Odessa National Academy of Telecommunications, Ukraine, in 2008 , and an associate professor diploma of the Switching Systems department from the Odessa National Academy of Telecommunications, Ukraine, in 2011. T. R. Shmeleva is the author of more than 80 papers and chapters published in journals, books, and conference proceedings. Her research interest is Petri net theory and its application in networking, especially in verifications of protocols, and performance evaluation. She developed an analysis of infinite Petri nets with regular structure and reenterable colored Petri net models of networks. Since 2005, she has been with the A.S. Popov Odessa National Academy of Telecommunications, where she is currently an Associate Professor in the Switching Systems Department. Tatiana is a member of SIAM.

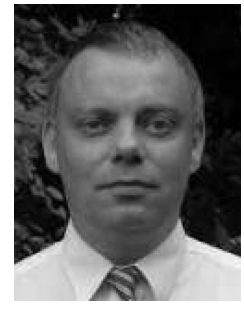

Jan Friso Groote is a Dutch computer scientist. Groote studied computer science at Twente University obtaining his master's degree in 1988 under supervision of prof. Ed Brinksma. He obtained his $\mathrm{PhD}$ thesis in 1991 under Prof. Jan Bergstra and Prof. Jos Baeten at the University of Amsterdam, while working at the Centre for Mathematics and Computer Science in Amsterdam. He contributed to structural operational semantics and verification technology. His particular contributions include the tyft/tyxt format for operational rules, the first algorithm to determine branching bisimulation and the cones and foci method to prove correctness of protocols and distributed algorithms. He is the founding father of the process modelling language and analysis tool sets muCRL and mCRL2. His current interest lies in the improvement of the quality of software by modeling via domain specific languages of which the quality is guaranteed using automatic verification. Since 1998 he has worked as a Full Professor in verification technology at Eindhoven University of Technology. He is a member of the ACM. 